\title{
A polyetic modelling framework for plant disease emergence
}

Article

Accepted Version

Willocquet, L., Savary, S., McDonald, B. A. and Mikaberidze, A. (2020) A polyetic modelling framework for plant disease emergence. Plant Pathology, 69 (9). pp. 1630-1643. ISSN 0032-0862 doi: https://doi.org/10.1111/ppa.13249 Available at https://centaur.reading.ac.uk/91045/

It is advisable to refer to the publisher's version if you intend to cite from the work. See Guidance on citing.

To link to this article DOI: http://dx.doi.org/10.1111/ppa.13249

Publisher: Wiley-Blackwell

All outputs in CentAUR are protected by Intellectual Property Rights law, including copyright law. Copyright and IPR is retained by the creators or other copyright holders. Terms and conditions for use of this material are defined in the End User Agreement.

\section{www.reading.ac.uk/centaur}

\section{CentAUR}

Central Archive at the University of Reading

Reading's research outputs online 


\section{A polyetic modelling framework for plant disease emergence}

3 Laetitia Willocquet $^{\mathrm{a1},{ }^{*}}$, S. Savary ${ }^{1}$, B.A. McDonald ${ }^{2}$, A. Mikaberidze $\mathrm{M}^{2,3}$

$5 \quad{ }^{1}$ AGIR, INRAE, Université de Toulouse, INPT, INP-EI Purpan, Castanet-Tolosan, France

6 2Plant Pathology, Institute of Integrative Biology, Zurich (IBZ), ETH Zurich, LFW B16, 8092 Zurich, Switzerland

7 '3 Current address: School of Agriculture, Policy and Development, University of Reading, Whiteknights, Reading,

8 RG6 6AR United Kingdom

9 * Corresponding author. Email: Laetitia.willocquet@inra.fr

10

11 Abstract

12 Plant disease emergences have dramatically increased recently as a result of global changes, especially with

13 respect to trade, host genetic uniformity, and climate change. A better understanding of the conditions and

14 processes determining epidemic outbreaks caused by the emergence of a new pathogen, or pathogen strain, is

15 needed to develop strategies and inform decisions to manage emerging diseases. A polyetic process-based

16 model is developed to analyse conditions of disease emergence. This model simulates polycyclic epidemics

17 during successive growing seasons, the yield losses they cause, and the pathogen survival between growing

18 seasons. This framework considers an immigrant strain coming into a system where a resident strain is already

19 established. Outcomes are formulated in terms of probability of emergence, time to emergence, and yield loss,

20 resulting from deterministic and stochastic simulations. An analytical solution to determine a threshold for

21 emergence is also derived. Analyses focus on the effects of two fitness parameters on emergence: the relative

22 rate of reproduction (speed of epidemics), and the relative rate of mortality (decay of population between

23 seasons). Analyses revealed that stochasticity is a critical feature of disease emergence. The simulations

24 suggests that: (1) emergence may require a series of independent immigration events before a successful 
25 invasion takes place; (2) an explosion in the population size of the new pathogen (or strain) may be preceded by

26 many successive growing seasons of cryptic presence following an immigration event, and; (3) survival between

27 growing seasons is as important as reproduction during the growing season in determining disease emergence.

KEYWORDS

disease emergence, process-based model, pathogen fitness, polyetic epidemics, pathogen survival

\section{INTRODUCTION}

33 The emergence of disease in plant populations has important impacts on both agricultural production and

34 natural ecosystems (Anderson et al., 2004; Lucas, 2017). While emerging plant diseases threaten biodiversity

35 and the entire range of services contributed by plants to the biosphere (Anderson et al., 2004), the emergence

36 of plant diseases constitutes an immediate threat to food security, from local to global scales, because of the

37 losses in production, and also because losses to plant disease affect food access (economic or physical) and the

38 quality of food (Savary et al. (2017). The literature provides growing evidence that plant disease emergences

39 have dramatically increased recently, as a result of global changes in trade, host genetic uniformity, and climate

40 (Anderson et al., 2004; Fisher et al., 2012; McDonald and Stukenbrock, 2016; Paini et al., 2016).

A relatively recent example of emergence of new pathogen strains is the introduction into Europe of a strain carrying the A2 mating type of Phythophthora infestans, the causal agent of potato late blight

43 (Zwankhuizen and Zadoks, 2002; Lucas, 2017). The emergence of this strain and its lineages, both resistant to 44 metalaxyl and more aggressive, led to more diversified, sexually reproducing, pathogen populations, and 45 increased disease intensity in Europe (Goodwin et al., 1996). Stem rust of wheat is another example. Stem rust epidemics, which were common in the USA during the first half of the last century, became rare after the pathogen (Puccinia graminis f. sp. tritici) was controlled by combining the deployment of new resistance genes 48 in wheat varieties with the eradication of barberry, which is the alternate host on which the pathogen 
49 reproduces sexually (Roelfs, 1978; 1985). In 1998, new races of this pathogen (called Ug99) were detected in

50 Uganda that were virulent against resistance genes present in wheat varieties widely grown in East Africa,

51 leading to local but severe epidemics in the region (Singh et al., 2015). International efforts to generate and

52 deploy resistant varieties helped to limit impacts from races of these new lineages (Singh et al., 2015), but the

53 recent detection of stem rust in different parts of Europe is now threatening wheat production in this part of

54 the world (Saunders et al., 2019). A third and recent example of strain introduction is that of Puccinia striiformis

55 f. sp. tritici, the causal agent of stripe (yellow) rust of wheat, into North-Western Europe in 2011 (de Vallavieille-

56 Pope et al., 2018) which caused serious epidemics.

An example of emergence of a new pathogen is Pyricularia graminis-tritici, the cause of wheat blast.

58 The disease was restricted to South America until 2016, when the pathogen was accidentally introduced and

59 caused a severe outbreak in South Asia (Ceresini et al., 2018). Another example of new pathogen emergence is

60 the Asian soybean rust, caused by Phakopsora pachyrhizi, which was introduced into South America at the

61 beginning of this century and has since severely impacted soybean production on that continent (Lucas, 2017).

62 Rhizomania is a virus disease of sugar beet that was first detected in the United Kingdom in 1987 and has since

63 spread, resulting in increasing numbers of epidemics (Gilligan et al., 2007). Other recent examples of disease

64 emergence with very disastrous impacts on perennial crops include huanglongbing on citrus in the New World

65 (Gottwald, 2010) and Xyllela fastidiosa on olive trees in Southern Europe (Saponari et al, 2019).

Disease emergence may be associated with changes in the environment, especially, human-made

67 changes. A much-debated example is the case of fusarium head blight of wheat (wheat scab), which has been

68 associated with the maize-wheat rotation, and with no-till practices (Zadoks and Schein, 1979; McMullen et al.,

69 2012). Another example is that of false smut of rice, which has been associated with the cultivation of hybrid

70 rice (Savary et al., 2017). A third example of environmental change-driven emergence is that of Sclerotium

71 rolfsii, a tropical pathogen on legumes (among many other hosts) becoming prevalent in the state of New York

72 as a result of warming climate (S. Pethybridge, Personal Communication). 
In their seminal article, Heesterbeek and Zadoks (1987) proposed a mathematical theory of pandemics,

74 with three phases: zero-order, first-order, and second-order epidemics. This theory considers two groups of processes, the spatial spread of disease and the accumulation of disease cycles within and across crop cycles, to analyse pandemics. While the zero-order epidemic is field-bound and polycyclic, the first-order epidemic is area-bound and polycyclic, and the second-order is both continental and polyetic. The present article is a response, some thirty years later, to this article. Figure 1 represents a synthesis of processes which may be associated with disease emergence, organised in three paths. Path 1 is the invasion of a new pathogen into an ecosystem, through introduction, establishment, and spread. Path 1 is exemplified by the wheat blast epidemic

81 in Bangladesh. Path 2 is the emergence of disease in response to environmental changes in an ecosystem,

82 where environmental changes lead to disease intensification, further leading to disease spread within entire

83 (agro)systems. Path 2 is illustrated by fusarium head blight of wheat or false smut of rice. Path 3 is the

84 emergence of new strains through evolutionary processes. Path 3 is illustrated by wheat stem rust in Sub-

85 Saharan Africa. Emergence paths may be combined. For instance, Paths 1 and 3 are combined in the potato late

86 blight epidemic of the 1990s in Western Europe; Paths 1, 2, and 3 are combined in the emergence of stripe rust

87 in Western Europe.

Similar to the emergence and re-emergence of infectious diseases in humans (Wilcox and Collwell,

89 2005), the emergence of plant diseases entails the consideration of biocomplexity, i.e., of complex systems,

90 where the biology of pathogens and hosts, their genetics, the changing environments - both natural and

91 human-made, and the social and economic structures (including plant health management systems) interact.

92 The present analysis does not address the biocomplexity of plant disease emergence as a whole, but rather

93 focuses on a fragment of Figure 1, with emphasis on Paths 1 (emerging pathogens) and 3 (emerging strains).

94 Elements of Path 2 (environmental change) are subsumed in the form of stochastic features of the modelling 95 work. 
Here we present a series of hypotheses underpinning the processes at play in disease emergence.

97 These hypotheses involve both demography (epidemiology) and population genetics as follows:

98 (1) from an epidemiological standpoint, emergence is a polyetic process, i.e., it is a process spanning several

99 consecutive crop seasons (Zadoks, 1974; Zadoks and Schein, 1979; Heesterbeek and Zadoks, 1987);

100 (2) this polyetic process is inherently stochastic because it entails random and abrupt changes in the pathogen

101 and host populations (Shaw, 1994). The process is also affected by random fluctuations in the environment

102 (Gilligan and Van den Bosch, 2008);

103 (3) an important determinant of successful emergence is the diversity in the population from which the

104 emerging pathogen originates. We assume the pathogen (or pathogen strain) to be sampled by chance in a

105 large genetic pool. The more diverse this pool, the higher the likelihood of fit to a given biological (hosts) and

106 physical setting (McDonald and Stukenbrock, 2016);

107 (4) pathogen migration (introduction) is often the primary mechanism associated with disease emergence

108 (McDonald and Stukenbrock, 2016);

109 (5) the level of crop losses associated with epidemics constitutes a useful metric for the impact of disease

110 emergence (Savary et al., 2006; 2017; 2019).

111 A range of models have been developed to analyse the dynamics of epidemics or pathogen populations

112 over multiple crop seasons. Leonard (1977) analysed the dynamics of plant pathogen genotypes over seasons

113 to investigate plant pathogen evolution under the gene-for-gene hypothesis. Since then, several polyetic

114 models have considered cycles of epidemic processes (disease transmission in the presence of the host)

115 followed by survival processes (pathogen decay in the absence of the host). Several models have considered

116 one pathogen genotype in order to address, e.g., thresholds for persistence according to epidemiological

117 parameters (e.g., Gubbins et al., 2000; Madden and Van den Bosch, 2002), whereby persistence corresponds to

118 disease emergence caused by invasion. These models were expanded to consider two pathogen genotypes to

119 analyse the evolutionary dynamics of pathogen populations (Van den Berg et al., 2011; Hamelin et al., 2011). 
120 Comparatively fewer stochastic polyetic models have been developed, showing chaotic polyetic patterns (Shaw,

121 1994), or guiding management strategies (with a spatially explicit stochastic model of sugar beet rhizomania;

122 Gilligan et al., 2007). To our knowledge, no model has yet been developed which simultaneously accounts for

123 polyetic processes, stochasticity, and the occurrence of several pathogen genotypes. Furthermore, none of the

124 polyetic models reported so far explicitly accounts for the impact of disease on yield loss.

125 The objectives of this work were to: (1) design a modelling framework to better define the conditions

126 determining disease emergence, (2) illustrate the use of the model by considering fitness components that

127 characterize the growth of the pathogen population during the growing season and its survival between

128 growing seasons and analysing their effects on disease emergence, and (3) draw some conclusions on

129 properties associated with disease emergence.

130

\section{2. MATERIALS AND METHODS}

132 2.1 Model requirements

133

134 Model specifications were established to build a structure incorporating processes related to population

135 genetics, epidemiology, and crop losses in order to analyse the conditions associated with disease emergence

136 and its effects on yield. The model and the outcomes of the analysis apply equally well to: (i) the situation when

137 a strain of a new pathogen is emerging on the background of another, resident pathogen population infecting

138 the same host, and (ii) the situation where a new, immigrant strain of a pathogen emerges on the background

139 of an already established population of the same pathogen. For the sake of simplicity, we will only refer to the

140 second situation below. The following requirements for the model were identified:

141 - Because disease emergence takes place over several crop seasons (Gilligan and Van den Bosch, 2008),

142 the model generates dynamics of epidemics and pathogen survival over successive cropping cycles, i.e., 

1987).

- Epidemics of many plant diseases entail secondary infections occurring during a crop season. The model therefore considers polycyclic epidemics within crop seasons.

- The model involves different strains of a pathogen in order to account for the evolutionary processes involved in disease emergence.

- Disease emergence often originates from the migration of a new pathogen (Fig. 1, Path 1), or of a new pathogen strain (Fig. 1, Path 3), into an agrosystem. The model therefore incorporates an immigration process.

- Modelling of the dynamics of primary inoculum with varying numbers of propagules, originating from preceding crop seasons and/or from immigration, and decaying over time, is a requirement, because (1) primary inoculum enables the initiation of seasonal epidemics, (2) a migrating pathogen strain enters the system as primary inoculum, and (3) primary inoculum also constitutes the link between two seasonal epidemics, and therefore provides the bridge needed to consider polyetic epidemics.

- Crop losses are an essential feature of epidemics in agroecosystems. The model therefore translates multi-seasonal, polyetic epidemics into their impact on crop performance as yield losses.

\subsection{Model description}

161 The system considered in the model is $1 \mathrm{~m}^{2}$ of a crop, under the "mean-field" hypothesis: the system 162 considered is surrounded by systems with the same features and dynamics. This $1 \mathrm{~m}^{2}$-system and its

163 surroundings are repeated in successive crop seasons separated by off-seasons. During any crop season, this

164 system and its neighbours are considered in a steady-state relationship. In particular, incoming and outgoing 165 inoculum between these systems cancel each other out, so that the net inflow/outflow balance is null. The 
166

167

168

169

170

171

172

173

174

175

176

177

178

179

180

181

182

183

184

185

186

187

188

model time step is one day, so as to accommodate processes which can have fast dynamics, such as polycyclic processes.

We consider a crop that is grown in regular cropping cycles (Supplementary Figure 1). Each cropping cycle consists of the period when the crop is present (crop season) and the period when the crop is absent (offseason). The crop season starts from crop planting and ends at harvest, and has two phases: the crop establishment phase and the crop growth phase (or, shortly, the growing season). The duration of each of the two phases (CEP, crop establishment period, and CGP, crop growth period) can vary depending on the crop, crop type (winter or spring crop) and location. Simulations start at crop planting, and are run for 30 cropping cycles.

The model considers one host plant genotype, e.g. a variety of a given crop, which can be infected by two strains of a given pathogen: a local (or resident) strain and an exogenous (or immigrant) strain. The local pathogen strain is present at the beginning of the simulation, while the exogenous pathogen strain is introduced into the system during the course of the simulation. The local population consists of strains that are already well adapted to local conditions. This local population is represented in the model by one local strain which has fixed demographic parameters. The exogenous population is established in a range of conditions (outside from the system), which may differ from the conditions of the considered system. This population is therefore more diverse, and generally less well adapted to the local conditions of the system. It thus consists of strains with a broader range of fitness attributes compared to the local population. This exogenous population is represented in the model by one strain with a fitness that can vary over cropping cycles. This variation reflects the hypothesis that the exogenous strain is less well adapted to the local conditions than the resident strain, and therefore is less well adapted to the environmental variations over cropping cycles. Each cropping cycle therefore involves two strains of the pathogen, the (fixed) local strain, and the (variable, random) exogenous strain. 

and Willocquet, 2014) in Figure 2. These are the processes involved in the development of epidemics, including

191 primary and secondary infections: RI (rate of infection); processes involved in the survival and decay of 192 inoculum: Rdecay (rate of inoculum decay); and processes involved in yield losses incurred from disease: $R L$ 193 (rate of loss). These processes are next described in greater detail. The model variables and parameters are 194 described in Table 1.

195 In each cropping cycle, the epidemic starts with primary infections (RPI), which take place at the end of 196 the crop establishment phase, as the crop growth phase starts. Primary infections have two origins. First, 197 primary infections can originate from inoculum produced from epidemics which took place in previous crop 198 seasons (polyetism), and second, primary infections can result from incoming inoculum (immigration from an exogenous population). In the beginning of the crop growth phase, the rate of primary infections for each

200 strain, $j=1$ (local), or 2 (immigrant), is therefore written as:

$201 R P I_{j}=\operatorname{convSP} \times S_{j}+R M_{j}$

202 Where convSP is the conversion of surviving inoculum into a rate of primary infections; $S_{\mathrm{j}}$ is the number of 203 surviving propagules for each strain; and $R M_{\mathrm{j}}$ is the rate of infections originating from immigrant strains, 204 referred to as the rate of immigration. The rate of primary infections, $R P I_{\mathrm{j}}$, has the value given by Eq. (1) only on 205 the first time step of each growing season and is set to zero at all other times. An epidemic takes place as the injury level, $i$, increases according to a logistic curve (exponential 207 increase of secondary infections, limited by the carrying capacity of the host crop) with a relative rate of 208 growth, RRg. As the seasonal epidemic unfolds, interaction between strains takes place, in the form of 209 competition towards host (crop) sites. This interaction between strains accounts for the maximum possible level 210 of injury (carrying capacity) at a given time, considering all plant sites occupied by the different strains at this 211 time. The rate of infection of each strain $j$, comprising primary and secondary infections, is therefore written as: 212 $R I_{\mathrm{j}}=\left[R R g_{\mathrm{j}} \times i_{\mathrm{j}} \times\left(1-\left(\left(i_{1}+i_{2}\right) /\right.\right.\right.$ imax $\left.\left.)\right)\right]+R P I_{\mathrm{j}}+$ starter $_{\mathrm{j}}$ 
213 where $R R g_{j}$ is the relative rate of injury increase for strain $j ; i_{j}$ is the injury level of strain $j ; i_{1}$ is the injury level

214 caused by the local strain; $i_{2}$ is the injury level caused by the immigrant strain; imax is the carrying capacity of

215 injury, i.e., the maximum level of injury; $R P I_{j}$ is the rate of primary infections associated with strain $j$; and starter $r_{j}$

216 is the number of primary infections at the beginning of the multiple-cropping cycle simulation (this parameter is

217 non-zero only during the first time step of the cropping cycle 1).

218 At the end of a cropping cycle, the terminal injury level $\left(i_{j}\right)$ is converted into surviving inoculum, $S_{j}$, for

219 each of the two strains. The number of surviving propagules decreases over time according to a negative

220 exponential dynamics, at a speed proportional to a relative rate of decay $\left(R R D_{\mathrm{j}}\right)$ :

221 Rdecay $_{\mathrm{j}}=R R D_{\mathrm{j}} \times S_{\mathrm{j}}$

222 where $R$ decay $y_{j}$ is the rate of decay of surviving propagules of strain $j ; R R D_{j}$ is the relative rate of decay of 223 surviving propagules of strain $j$, and $S_{j}$ is the number of surviving propagules of strain $j$.

224 Injuries impair the physiological processes involved in crop growth and yield build-up, ultimately 225 leading to yield losses. The several possible damage mechanisms from injuries are represented in a very 226 simplified manner by a single rate of yield loss, $R L$, which increases proportionally to the running level of 227 combined injuries caused by both strains, $i_{1}+i_{2}$ :

$228 R L=R R L \times\left(i_{1}+i_{2}\right) \times[1-(Y L / Y a)]$

229 where $R R L$ is the relative rate of yield loss; $i_{1}$ and $i_{2}$ are the injury levels from the local and immigrant strains,

230 respectively; $Y L$ is the yield loss, i.e. the yield reduction from a disease-free attainable yield; and $Y a$ is the 231 attainable yield, i.e., the yield level in the absence of disease. At the end of each crop growth phase, yield loss is 232 reset to zero, so that the new cropping cycle starts without losses. 
237 Initial values are zero for all state variables ( $i, S$, and $Y L$ ). Parameters dimensions and values are listed in Table 1.

238 The durations of the crop establishment period (CEP) and of the crop growth period (CGP) are both set to 120

239 days, representing, for example, approximate durations for a winter wheat crop grown in a temperate region of

240 the world. An epidemic of the local strain is initiated at the end of CEP in the first cropping cycle with an initial

241 value (starter; Table 1) for the local strain of 0.01 day $^{-1}$. The conversion of surviving propagules into a rate of

242 primary infections (convSP) is set to 0.01 , meaning that for example 100 surviving entities are translated into 1

243 primary infection during the first time step of CGP. The carrying capacity for injury level, imax, is set to 100 in

244 order to generate injury levels expressed as percent. In the same way, Ya, the attainable yield, is set to 100 in

245 order to generate yield losses expressed as percent.

$246 R R L$ is set to 0.05 , meaning that combined disease injury $\left(i_{1}+i_{2}\right)$, when at low levels, entails an increase

247 in yield loss at each time step which corresponds to $5 \%$ of the level of disease injury. $R R g_{1}$ and $R R D_{1}$ values are

248 set to 0.07 and 0.01 , respectively.

249

2502.4 Model analyses: conditions of emergence of an immigrant strain

$251 \quad 2.4 .1$ Framework of analyses

252 We consider a pathosystem with two pathogen strains: a local (resident), and an immigrant (exogenous) strain.

253 The fitness of each of the two strains is represented by two essential components: the ability to reproduce

254 during the growing season [represented by a relative, or intrinsic, rate of growth, $R R g_{j}$ in Equation (2)] and the

255 rate of population decay [represented by a relative, or intrinsic, rate of decay, $R R D_{j}$ in Equation (3), Table 1], the

256 latter characterizing the ability of a pathogen strain to survive in the absence of host plants. As a convention,

257 the subscript $j=1$ refers to a local strain and $j=2$ refers to an immigrant strain. Fitter strains reproduce faster on

258 the host during the growing season and decay more slowly over time.

259 We addressed the question of emergence of immigrant strains as follows. A given agroecosystem

260 harbours a resident diversity of strains; however, all these strains are assumed to be equally adapted to the 
considered agroecosystem - i.e. they have similar fitness. As a simplification, the entire population of resident strains in an agroecosystem is therefore represented by one strain, exhibiting two central values for $R R g_{1}$ and

$263 R R D_{1}$. Because these local populations are assumed to be established and in a dynamic equilibrium, we further 264 assume no variation over time for parameters $R R g_{1}$ and $R R D_{1}$. In the absence of immigration, successive epidemics occur in the considered agroecosystem. These epidemics consist of overlapping disease cycles (polycyclic epidemics), and each epidemic results from the carry-over of inoculum from a previous epidemic that took place in the previous crop seasons. The resulting pattern of disease over successive crop seasons (polyetic process) in an agroecosystem thus results from the concatenation of successive (polycyclic) epidemics. In order to investigate conditions for emergence, we consider an immigrant strain, which originates from a very large pool of possible strains. In a first (deterministic) regime, the fitness parameters of the 271 immigrant strain, $R R g_{2}$ and $R R D_{2}$, are assumed to be constant throughout the successive simulated cropping seasons. In a second (stochastic) regime, the fitness parameters of the immigrant strain are drawn at random from a normal distribution with central values $R R g_{2}$ and $R R D_{2}$, and with variation about these values. This drawing is made at the beginning of each cropping cycle, and the values drawn are kept constant within each cropping cycle. This stochastic regime reflects the hypothesis of a strain which is not well adapted to the local environment, with a fitness that varies as environmental conditions vary over cropping cycles. The execution of the model over a succession of 30 cropping cycles is referred to as a simulation. We investigated a scenario in which the immigrant strain is introduced once, at cropping cycle 10, at the beginning of the growing season. This way, the immigrant strain is introduced into a stabilized system where the local 280 strain is already established. We used the simulation model (Section 2.2, Figure 2) to study two dynamic 281 regimes: (i) a deterministic regime, in which $R R g_{2}$ and $R R D_{2}$ had fixed values during a given simulation (section 282 2.4.2 below), and (ii) a stochastic regime, in which the values of either $R R g_{2}$ or $R R D_{2}$ or both were drawn from a 283 normal (Gaussian) distribution at the beginning of each cropping cycle and kept at these values during each 284 cropping cycle (section 2.4.3 below). We also derived approximate analytical expressions for the thresholds of 
285

286

emergence of the immigrant strain by representing the simulation model as a discrete time map and investigating its linear stability (Section 2.4.4 below and Appendix A).

The outcomes of the analyses were synthesised according to three features characterising disease emergence of an immigrant strain and its consequences: the probability of emergence, the time to emergence, and the yield loss associated with the emergence. We consider that the immigrant strain has emerged if it exceeds the resident strain in terms of its AUDPC (area under disease progress curve, i.e., the accumulated injury incurred within a growing season) during at least three cropping cycles after its introduction. The probability of emergence, $P_{\text {emerg }}$, was estimated as the proportion of simulations that resulted in emergence. In each individual simulation that resulted in emergence, the time to emergence, $T_{\text {emerg, }}$ was defined as the number of cropping cycles between the introduction of the immigrant strain and the first cropping cycle when the AUDPC of the immigrant strain exceeded that of the resident strain. To quantify yield loss in each simulation, we calculated the average yield losses caused by both the resident and the immigrant pathogen strains over the 30 cropping cycles.

The model was developed using the Stella software (STELLA Architect version 1.1.2) and subsequently translated to the Python programming language (version 3.4.3), where the bulk of the analysis was conducted. The system of Equations (1)-(4) was solved and analysed using Python packages numpy (version 1.13.3) and scipy (version 1.0.0), and the figures were produced using the Python package matplotlib (version 2.1.1). Parts of the analytical investigation were performed with Wolfram Mathematica (version 10.3 for Linux).

\subsubsection{Deterministic approach}

We performed three sets of simulations in order to analyse the individual effects of $R R g_{2}, R R D_{2}$, and the combined effects of $R R g_{2}$ and $R R D_{2}$ on disease emergence.

A first analysis was conducted to address conditions of emergence associated to $R R g_{2}$. In this first analysis, 100 simulations were run with $R R g_{2}$ increasing from 0.06 to $0.12 \mathrm{day}^{-1}$ with a constant increment of 
$309 R R g_{2}$ between simulations, while $R R D_{2}$ was fixed $\left(0.02\right.$ day $\left.^{-1}\right)$. The $R R D_{2}$ value chosen corresponds to the

310 hypothesis of an immigrant strain with a lower survival capacity than the resident strain $\left(R R D_{1}=0.01\right.$ day $\left.^{-1}\right)$. In

311 the second analysis, we assessed conditions of emergence according to $R R D_{2}$. Here, 100 simulations were run

312 with $R R D_{2}$ increasing from 0 to $0.05 \mathrm{day}^{-1}$ with a constant increment between simulations, while $R R g_{2}$ was fixed

$313\left(0.1 \mathrm{day}^{-1}\right)$. This $R R g_{2}$ value corresponds to the hypothesis of an immigrant strain with a higher aggressiveness

314 than the resident strain $\left(R R g_{1}=0.07\right.$ day $\left.^{-1}\right)$. In a third analysis, both $R R g_{2}$ and $R R D_{2}$ were considered with respect

315 to emergence. $R R g_{2}$ and $R R D_{2}$ were varied in the same ranges as in the first and second analyses over a total of

$31610^{4}$ simulations (100 x 100 runs).

\section{2.4.3. Stochastic approach}

319 As in the deterministic approach, the individual effects of $R R g_{2}, R R D_{2}$, and combined effects of $R R g_{2}$ and $R R D_{2}$ 320 were subsequently analysed.

321 To address conditions of emergence associated to $R R_{2}, 100$ sets of simulations were executed with 322 fixed $R R g_{2}$ values ranging from 0.06 to 0.12 day $^{-1}$, with a constant increment. For each $R R g_{2}$ value considered 323 (i.e., for each set of simulations), 5000 stochastic runs were executed, within which the values of $R R D_{2}$ were 324 drawn at the beginning of each cropping cycle as random numbers from the normal (Gaussian) distribution with 325 the mean 0.02 day $^{-1}$ and the standard deviation 0.007 day $^{-1}$. These $R R D_{2}$ values then remained constant during

326 the whole cropping cycle until the beginning of the next growing season, when a new random value was 327 chosen.

The second analysis was conducted in the same way as the first analysis, but focused on $R R D_{2}: 100$ sets

329 of simulations were executed with fixed $R R D_{2}$ values ranging from 0 to 0.05 day $^{-1}$, with a constant increment.

330 For each $R R D_{2}$ value considered, 5000 stochastic runs were executed, within which the values of $R R g_{2}$ were 331 drawn at the beginning of each cropping cycle as random numbers from the normal distribution with mean 0.1 
332

day $^{-1}$ and standard deviation 0.035 day $^{-1}$. These $R R g_{2}$ values then remained constant during the whole cropping cycle.

In a third analysis, the values of both $R R g_{2}$ and $R R D_{2}$ were drawn from the normal distribution at the beginning of each cropping cycle with means ranging from 0.05 to 0.12 day $^{-1}$ for $R R G_{2}$, and ranging from 0 to 0.04 for $R R D_{2}$, and with standard deviations constituting a constant proportion, 0.35 , of the corresponding mean values. As in the previous analyses, $R R g_{1}$ and $R R D_{1}$ values remained constant within each cropping cycle. We ran 200 stochastic realizations for each point of the $100 \times 100$ grid of $R R g_{2} \times R R D_{2}$ values considered.

\subsubsection{Analytical approach}

The overall fitness of the pathogen strain $j$ is given by its polyetic (or multi-season) basic reproductive number (see Appendix A for the derivation):

$R_{0 \mathrm{p}, \mathrm{j}}=\operatorname{convSP} \times \exp \left[\left(R R g_{\mathrm{j}} \times \mathrm{T}_{\mathrm{s}}\right)-\left(R R D_{\mathrm{j}} \times T_{\mathrm{BS}}\right)\right]$

where $T_{\mathrm{S}}$ is the crop growth duration $\left(T_{\mathrm{S}}=C G P\right)$ and $T_{\mathrm{BS}}$ is the delay between two successive growing seasons.

The index "p" in $R_{0 \mathrm{p}}$ refers to "polyetic", in order to distinguish $R_{0 \mathrm{p}}$ from $R_{0}$, which usually refers to the "within season" basic reproductive number in the epidemiological literature (e.g., Zadoks and Schein, 1979; Anderson and May, 1986; Campbell and Madden, 1990). Biologically, $R_{0 \mathrm{p}, \mathrm{j}}$ represents the number of units of crop injury appearing at the beginning of a given growing season following the introduction of a unit host injury in the beginning of the previous growing season. $R_{0 \mathrm{p}, \mathrm{j}}$ incorporates both the ability of a strain $j$ to multiply during crop growth and to survive between growing seasons. Hence, in the exponent of Eq. (5), the two components of pathogen fitness, $R R g_{j}$ and $R R D_{j}$, are weighted by $T_{\mathrm{S}}$ and $T_{\mathrm{BS}}$, respectively.

As in the previous analysis, we consider the situation when the local pathogen strain is viable when present alone: its reproduction during the growing season exceeds its losses between growing seasons, i.e., $R_{0 \mathrm{p}, 1}>1$. In this case, the immigrant strain will emerge if its polyetic basic reproductive number exceeds the polyetic basic reproductive number of the local strain, i.e., $R_{0 \mathrm{p}, 2}>R_{0 \mathrm{p}, 1}$. The emergence threshold corresponds to 
$356 R_{0 \mathrm{p}, 2}=R_{0 \mathrm{p}, 1}$. We solve this equation with respect to $R R g_{2}$ and obtain the threshold value of $R R g_{2}$ above which

357 emergence takes place:

$358 R R g_{2, \text { thresh }}=\left[\left(T_{\mathrm{BS}} / T_{\mathrm{S}}\right) \times\left(R R D_{2}-R R D_{1}\right)\right]+R R g_{1}$

359 Similarly, the emergence threshold can be expressed in terms of $R R D_{2}$ :

$360 R R D_{2, \text { thresh }}=\left[\left(T_{S} / T_{B S}\right) \times\left(R R g_{2}-R R g_{1}\right)\right]+R R D_{1}$.

361 Here, the immigrant strain emerges when its relative rate of decay is below the threshold, i.e., $R R D_{2}<R R D_{2, \text { thresh }}$

362 Eqs. (6) and (7) were derived under the assumption that the saturation effects of the logistic growth are

363 negligible. This is justified at sufficiently low relative rates of growth for each of the strains $R R g_{j}$, and at short

364 enough CGP, so that the host tissue does not become a limiting factor for any of the two pathogen strains. Note

365 that the simulation model in Sec. 2.2 does not make this approximation. See Appendix A for more mathematical 366 details.

\section{3. RESULTS}

3693.1 An example of dynamics of crop injuries and losses simulated with the stochastic approach

Figure 3 displays examples of simulated dynamics using fitness parameters for the immigrant strain drawn from

371 a normal distribution at the beginning of each cropping cycle, the parameter values remaining fixed within a

372 given cropping cycle. Means of $R R g_{2}$ and $R R D_{2}$ are equal to the values used for the local strain $(0.07$ for $R R g$ and

3730.01 for $R R D$ ) and their standard deviations are 0.03 and 0.003 , respectively. The three top panels display injury

374 dynamics leading to (1) non-emergence of the immigrant strain (Fig. 3a), (2) co-occurrence of both strains

375 where the predominant strain varies over cropping cycles (Fig. 3b), and (3) rapid emergence of the immigrant

376 strain (Fig. 3c). Because parameters are fixed for the local strain, simulation leading to non-emergence (Fig. 3a)

377 shows an equilibrium state with a maximum level of injury which reaches a constant value starting from the $6^{\text {th }}$

378 cropping cycle. When both strains co-exist (Fig. 3b), the stochasticity of $R R g_{2}$ and $R R D_{2}$ produces a large

379 variation over cropping cycles in both injury level and the respective frequency of each strain. In the case of 
380

381 $382 R R D_{2}$.

rapid emergence of the immigrant strain (Fig. 3c), the immigrant strain very quickly overcomes the local strain, but displays a large variation in disease intensity over cropping cycles, because of stochasticity in $R R g_{2}$ and

$$
\text { Simulated yield loss, primary inoculum, and relative rates of growth and decay corresponding to the }
$$
example of a rapidly emerging strain (Fig. 3c) are displayed in Figure 3d-f. Yield losses vary over time (Fig. 3d), with a pattern similar to that observed for injury dynamics (Fig. 3c). At the end of each crop season, the terminal disease injury from each strain is proportionally converted to surviving propagules. The number of surviving propagules then decays exponentially over time and constitutes the primary inoculum for the subsequent growing season (Fig. 3e). This primary inoculum translates into primary infections at the beginning of each crop growth phase (Fig. 3e). Relative rates of growth and of decay of the immigrant strain vary over cropping cycles, while remaining constant within each cropping cycle (Fig. 3e). These stochastic values of $R R g_{2}$ and $R R D_{2}$ are driving the dynamics of injury (Fig. 3c) and of primary inoculum (Fig. 3e) over cropping cycles.

\subsection{Individual effects of the relative rates of epidemic growth and inoculum decay on disease emergence}

When we consider $R R g_{2}$ variation in the deterministic regime, $P_{\text {emerg }}$ rises suddenly from zero to one as the $R R g_{2}$ value is increased (Fig. 4a, blue curve). The reason is that the immigrant strain can only emerge if it is able to grow fast enough during the growing season. More fit immigrants, when they emerge (i.e., when $P_{\text {emerg }}=1$ in Fig. 4a), emerge more rapidly as $R R g_{2}$ increases: $T_{\text {emerg }}$ decreases monotonically as we increase the immigrant strain's fitness by increasing $R R g_{2}$ (Fig. 4c, blue curve). As we increase $R R g_{2}$, the amount of disease caused by the immigrant strain increases and so does the average yield losses incurred by both the resident and the immigrant pathogen strains (Fig. 4e, blue curve). Below the emergence threshold (Fig. $4 \mathrm{a}, R R g_{2}$ values for which $P_{\text {emerg }}=0$ ), the immigrant strain is absent, therefore the yield losses are only incurred by the resident strain, and are not affected by $R R g_{2}$. The analytical approach yields a threshold for emergence of $R R g_{2}$ at 0.09 day $^{-1}$ (Fig. 4 a, 
grey line), that is, slightly smaller than the threshold derived from the deterministic approach (Fig. 4a, blue

404 line).

When we include stochasticity, the transition between parameter areas of "no emergence" and

"emergence" is now gradual: $P_{\text {emerg }}$ increases continuously as $R R g_{2}$ values of the immigrant strain are increased

407 (red curve in Fig. 4a). Time to emergence also exhibits a different pattern in the stochastic regime. Emergence

408 starts at $R R g_{2}$ values much smaller than in the deterministic regime (Fig. 4c, red curve) with relatively small

409 values of $T_{\text {emerg }}$ (about 2 cropping cycles), then $T_{\text {emerg }}$ increases gradually, plateaus at about four cropping cycles,

410 and then declines with a curve close to, but above, that generated from the deterministic approach. Yield losses

411 show a similar pattern in the deterministic and stochastic regimes (compare red and blue curves in Fig. 4e),

412 although yield losses are somewhat lower in the stochastic regime than in the deterministic regime.

413 The effect of $R R D_{2}$ on emergence characteristics (Fig. 4b, d, f) mirrors the effect of $R R g_{2}$ (Fig. 4a, c, e),

414 because as fitness of the immigrant strain increases with $R R g_{2}$, it decreases with $R R D_{2}$. Under the deterministic

415 regime, $P_{\text {emerg }}$ drops abruptly from one to zero as $R R D_{2}$ is increased (Fig. $4 \mathrm{~b}$, blue curve): the immigrant strain

416 cannot emerge if its population decays too fast between growing seasons. In the same way, less fit immigrants

417 emerge more slowly, when they do emerge: $T_{\text {emerg }}$ increases monotonically as we reduce the immigrant strain's

418 fitness as $R R D_{2}$ increases (Fig. $4 \mathrm{~d}$, blue line). Average yield losses decrease as $R R D_{2}$ increases (Fig. $4 \mathrm{f}$ ), and

419 remain stable when $R R D_{2}$ values are above the threshold for emergence. The threshold for emergence

420 generated from the analytical approach, $R R D_{2}=0.025$, is slightly lower than the threshold generated from the

421 deterministic approach (Fig. 4b).

422

When we include stochasticity, $P_{\text {emerg }}$ diminishes continuously as $R R D_{2}$ is increased; time to emergence,

$423 T_{\text {emerg }}$, increases initially (with values slightly larger than those obtained from the deterministic approach),

424 reaches a maximum around the emergence threshold and gradually declines to small values. Yield losses show

425 a qualitatively similar pattern in the deterministic and stochastic regimes. As when investigating the effect of

$426 R R g_{2}$, yield losses are lower in the stochastic regime than in the deterministic regime (Fig. $4 \mathrm{f}$ ). 
4283.3 Combined effects of the relative rates of epidemic growth and inoculum decay on disease emergence

429 When using the deterministic approach, emergence and no emergence domains are clearly separated by a

430 straight line (Fig. 5a). This line reflects the abrupt transition from emergence to no emergence, as illustrated in

431 Figs. $4 \mathrm{a}$ and $4 \mathrm{~d}$. The domain of emergence corresponds to pairs of values of $R R g_{2}$ and $R R D_{2}$ below which

432 emergence takes place: for a given value of $R R g_{2}$, emergence will occur within a range of values of $R R D_{2}$ below a

433 given threshold. The dashed lines in Fig. 5a display the outcomes for fitness values used which correspond to

434 that of the resident strain. At $R R g_{2}=R R g_{1}=0.07$, emergence occurs for $R R D_{2}$ values slightly smaller than $R R D_{1}$.

435 Similarly, at $R R D_{2}=R R D_{1}=0.01$, emergence occurs for $R R g_{2}$ values that are slightly larger than $R R g_{1}$. The solid

436 grey line represents the analytical expression for the emergence threshold in terms of $R R D_{2}$, according to

437 Equation (7). That is, a line with slope $\left(T_{S} / T_{B S}\right)$ which equals 0.5 in our case, and an ordinate at origin of -0.025.

438 When stochasticity is included in the model, the transition between parameter domains of emergence

439 and no emergence becomes gradual (Fig. 5b). This gradual transition is a generalisation of the gradual change in

440 emergence probability according to $R R g_{2}$ and $R R D_{2}$ illustrated in Figs. $4 \mathrm{a}$ and $4 \mathrm{~d}$, respectively. As in Fig. $5 \mathrm{a}$, the

441 dashed black line represents the fitness parameters of the resident stain. In the same way, the parameter

442 region explored in Fig. $4 \mathrm{a}$ and $4 \mathrm{~b}$ is materialised with the yellow lines which refer to $R R g_{2}=0.1$ and $R R D_{2}=0.02$.

443 The most important effect of stochasticity is that emergence occurs at ranges of parameters that are below the

444 emergence threshold, where no emergence was possible according to the deterministic approach (within the

445 white area in Fig. 5a). Even if the immigrant strain is on average less fit than the local strain (i.e., in terms of its

446 average fitness components $R R g_{2}<R R g_{1}, R R D_{2}>R R D_{1}$ ), there is still a non-zero probability for its emergence.

447 This scenario corresponds to the region in Fig. $5 \mathrm{~b}$ above the black horizontal line and to the left from the black

448 vertical line. 


\section{DISCUSSION}

$452 \quad 4.1$ Key findings

453 The modelling framework which was designed in this work enables analysing the conditions underlying disease 454 emergence. According to our pre-set specifications, the model includes polyetism, stochasticity, and yield loss.

455 The analyses conducted here allow identifying important features associated with disease emergence.

A major finding is that stochasticity can be an important boundary condition for disease emergence.

Emergence reflects important changes in the status of a system (here crop health) that can be caused by rare events (e.g., Paini et al., 2016), associated with small sizes of immigrant or mutant subpopulations that initiate the process, and by polyetic processes that lead to significant reductions in population size between growing seasons (Shaw, 1994). Stochasticity associated with genetic factors such as bottlenecks and genetic drift is known to play an important role in the evolution of a host-pathogen interaction (McDonald, 2004). Stochasticity can also be introduced by environmental factors such as climatic conditions, which can differentially affect the fitness of strains in pathogen populations (e.g., Gilligan and Van den Bosch, 2008). In the present study, we focused on the latter, environmentally-induced, stochasticity. den Bosch, 2002) under a deterministic framework, leading to identification of thresholds for persistence. even when an immigrant strain is drawn from a population which is, on average, less fit than the local strain,

469 the immigrant strain may nevertheless emerge due to stochasticity; and conversely (2) even when an immigrant 470 strain is drawn from a population which is, on average, more fit than the local strain, the immigrant strain will 471 not necessarily emerge, and may face extinction. An important implication of this finding is that emergence 472 may require a series of independent immigration events involving new pathogen strains before a successful 473 invasion takes place. In some cases, a pathogen that appears to have suddenly emerged over the course of only 474 1-2 cropping cycles may have been present at a low level for decades before the proper conditions (e.g. 
conducive weather conditions) occurred to enable an explosion in population size and an observed

476 "emergence". This has important implications to guide future research, both in terms of modelling and

477 experimentation, and potentially to inform policy on emerging diseases.

Another important finding from our analyses is that survival between growing seasons is as important abilities and lacking durable survival structures such as chlamydospores, sclerotia or oospores are expected to undergo large bottlenecks between host growing seasons that will purge genetic diversity and increase the probability that less well-adapted immigrants occurring at lower frequencies will go extinct between growing seasons. Conversely, pathogens that compete well as saprophytes and/or produce long-lived survival structures will maintain high effective population sizes that sustain high levels of genetic diversity across growing seasons, enabling persistence of immigrants and novel mutants for long periods of time, even if they are less well adapted, and increasing the probability that these immigrants, mutants or recombinants can make a successful invasion. Although the importance of the survival phase has been recognized in earlier work (e.g., Heesterbeek and Zadoks, 1987; Gubbins et al., 2000; Madden and Van den Bosch, 2002, Hamelin et al., 2011), survival has often been overlooked by plant pathologists. Conversely, $R R g$ can be seen as the apparent infection rate of Van der Plank ( $r_{L}$; Campbell and Madden, 1990), for which ranges have been measured from disease progress curves

491 in many instances. The $R R g$ ranges explored in our analyses ( 0.05 to 0.12 ) fit well within ranges measured for epidemics of annual crop diseases (Kranz, 2003).

494 4.2 Comparing outcomes from the deterministic, analytical, and stochastic approaches

495 There is a good agreement between the analytical emergence thresholds (Eq. (6) and (7)) and the numerical 496 thresholds in the deterministic regime, although the threshold for emergence with respect to $R R g$ is slightly 497 lower when derived from the analytical approach as compared to the deterministic approach (Figs. 4, 5a), while 498 the opposite pattern is obtained for RRD (Figs. 4b, 5a). This difference can be explained by the limited duration 
499 (30 cropping cycles) of the numerical simulations. In some cases, the immigrant strain would be able to emerge,

500 but this would require more than 30 cropping cycles. On the contrary, the analytical threshold does not restrict

501 the number of cropping cycles and therefore generates thresholds for emergence that can occur over an infinite

502 time span. This explanation was confirmed by performing additional simulations conducted using the same

503 design that generated Figure 4, but including many additional cropping cycles (200). In that case, the agreement

504 between the two thresholds (from deterministic simulations and from analytical expressions) was perfect. In

505 future analyses using this framework, the threshold values to consider (from deterministic or analytical

506 approaches) will depend on the modelling objectives and the applications under consideration.

When investigating the probability of emergence, fitness thresholds are derived from the deterministic

508 approach, while such thresholds do not materialize in the stochastic approach because the emergence

509

probability can take values between 0 and 1 . The stochastic approach allows a strain with a fitness ( $R R g$ or $R R D$ )

510 mean value below the deterministic emergence threshold (i.e., lower values for $R R g$ and larger values for $R R D$ )

511 to emerge, with a probability which progressively declines as the mean fitness value moves away from the

512 threshold.

513 The time to emergence progressively declines as the fitness values increase in the deterministic

514 approach because it requires progressively less time for the immigrant strain to outcompete the resident strain.

515 Under the stochastic regime, a different pattern is exhibited, with the time for emergence increasing, reaching a

516 maximum, and eventually declining as the fitness values increase. This pattern can be interpreted as follows: at

517 low average fitness, the only way to achieve emergence in the stochastic regime is when high fitness values

518 from the tail of the distribution are drawn over several consecutive growing seasons, representing particularly

519 "lucky" realizations. There is a small proportion of such realizations (reflected by the small probability of

520 emergence), as they correspond to quite rare events, but when they do happen, emergence occurs relatively

521 fast. In contrast, at higher average fitness, there can be many other paths to emergence including those

522 realizations in which high fitness values appear sporadically, not necessarily in several consecutive seasons, 
523 leading to slower emergence on average. Thus, the two competing effects, (i) longer emergence due to reduced

524 mean fitness of the immigrant strain in the range of high fitness values and (ii) the preferential emergence of

525 only "lucky", "fast-emerging" realizations in the range of low fitness values, lead to emergence time reaching a

526 maximum in the stochastic regime.

527 Yield losses derived from the stochastic regime are lower than yield losses derived from the

528 deterministic approach (Figs. 4e, f). This difference can be seen as the consequence of differences observed

529 between these regimes both in terms of the probability of emergence (Figs. $4 a, b$ ) and the time to emergence

530 (Figs 4c, d): above the deterministic threshold, there are cases where disease does not emerge, or where time

531 to emergence is delayed in the stochastic approach, and therefore yield losses are not as high as in the

532 deterministic approach.

533

534 4.3 Further questions to address on disease emergence

535 Our analyses provide a series of elements to better understand how disease emerges. The model structure

536 allows addressing other important questions on disease emergence, such as:

537 - the effect of primary infection patterns on emergence: in the analyses we conducted, we considered only one

538 type of primary infection, as a single immigration event occurring at a single point in time. The model allows the

539 consideration of other patterns, including varying size of immigrant inoculum, or repeating inflows of immigrant

540 strains over several cropping seasons (instead of during only one cropping season).

541 - the immigration rate simply considers the entry into the system of a new strain, with no specific hypothesis

542 attached to the origin of this strain. The model also allows consideration of other potential sources of new

543 strains, including recombinants or mutants, which could originate from inside or outside of the zone of

544 emergence. 
545 - the model can also include adaptation of the pathogen population, for example by varying $R R g$ and $R R D$ over

546 time, or draw new parameters at the start of each cropping cycle according to the parameter values of the

547 preceding cropping cycle (Figure 1, path 3).

548 - the effect of variation of RRg within the growing season can be analysed in order to mimic the effect of

549 weather (e.g. warmer winters or drier summers) on epidemics and emergence (Figure 1, Path 2).

550 - the analyses were conducted with a relatively limited number of cropping cycles of simulation. This was

551 appropriate because a large amount of inoculum was used in the simulations. When considering a low rate of

552 immigration within a stochastic regime, much longer time frames may be needed to detect emergence.

553 - the effect of climate change on disease emergence can be addressed with this model by incorporating a

554 directional change in the mean and/or the standard deviation of some fitness parameters (e.g., $R R g$ and $R R D$ )

555 over successive cropping cycles.

556

Plant disease emergence is a complex phenomenon, with many system- and context-specific variants.

558 This work addresses the phenomenon in a simplified manner in order to derive some of its main features.

559 While this work needs to be continued, we hope that the present analysis provides a useful step towards

560 implementing more effective policies to prevent or delay plant disease emergence.

562 DATA AVAILABILTY STATEMENT

563 The data that support the findings of this study are available from the corresponding author upon reasonable 564 request. 


\section{REFERENCES}

566 Anderson, P. K., Cunningham, A.A., Patel, N.G., Morales, F.J., Epstein, P.R. and Daszak, P. (2004) Emerging

567 infectious diseases of plants: pathogen pollution, climate change and agrotechnology drivers. Trends in

$568 \quad$ Ecology and Evolution, 19, 535-544.

569 Anderson, R.M. and May, R.M. (1986) The invasion, persistence and spread of infectious diseases within animal

570 and plant communities. Philosophical Transactions of the Royal Society of London B, Biological Sciences, 314,

$571 \quad 533-570$.

572 Campbell, C.L. and Madden, L.V. (1990) Introduction to Plant Disease Epidemiology. New York, USA: John Wiley 573 and Sons.

574 Ceresini, P.C., Castroagudín, V.L., Rodrigues, F.Á., Rios, J.A., Aucique-Pérez, C.E., Moreira, S.I. et al. (2018) Wheat 575 blast: past, present, and future. Annual Review of Phytopathology, 56, 427-456.

576 Fisher, M.C., Henk, D.A., Briggs, C.J., Brownstein, J.S., Madoff, L.C., McCraw, S.L. and Gurr, S.J. (2012) Emerging 577 fungal threats to animal, plant and ecosystem health. Nature, 484, 186-194.

578 Forrester, J.W. (1961) Industrial Dynamics. Cambridge, Mass., USA: M.I.T. Press.

579 Gilligan, C.A. and Van den Bosch, F. (2008) Epidemiological models for invasion and persistence of pathogens.

$580 \quad$ Annual Review of Phytopathology, 46, 385-418.

581 Gilligan, C.A., Truscott, J.E. and Stacey, A.J. (2007) Impact of scale on the effectiveness of disease control 582 strategies for epidemics with cryptic infection in a dynamical landscape: an example for a crop disease. 583 Journal of the Royal Society Interface, 4, 925-934.

584 Goodwin, S.B., Sujkowski, L.S. and Fry, W.E. (1996) Widespread distribution and probable origin of resistance to 585 metalaxyl in clonal genotypes of Phytophthora infestans in the United States and western Canada.

$586 \quad$ Phytopathology, 86, 793-799.

587 Gottwald, T.R. (2010) Current Epidemiological Understanding of Citrus Huanglongbing. Annual Review of 588 Phytopatohlogy, 48, 119-39. 
589 Gubbins, S., Gilligan, C.A. and Kleczkowski, A. (2000) Population dynamics of plant-parasite interactions:

590 thresholds for invasion. Theoretical Population Biology, 57, 219-233.

591 Hamelin, F.M., Castel, M., Poggi, S. and D. and Mailleret, L. (2011) Seasonality and the evolutionary divergence 592 of plant parasites. Ecology, 92, 2159-2166.

593 Heesterbeek, J.A.P. and Zadoks, J. C. (1987) Modelling pandemics of quarantine pests and diseases: problems 594 and perspectives. Crop Protection, 6, 211-221.

595 Kranz, J. (2003) Comparative Epidemiology of Plant Diseases. Berlin, Germany: Springer.

596 Leonard, K.J. (1977) Selection pressures and plant pathogens. Annals of the New York Academy of Sciences, 287, $597 \quad 207-222$.

598 Lucas, J.A. (2017) Fungi, food crops, and biosecurity: advances and challenges. In: Advances in Food Security 599 and Sustainability. Amsterdam, The Netherlands: Elsevier, pp. 1-40.

600 Madden, L.V., Van Den Bosch, F. (2002) A population-dynamics approach to assess the threat of plant pathogens 601 as biological weapons against annual Crops. BioScience, 52, 65-74.

602

603

604

605

606

McDonald, B.A. (2004) Population Genetics of Plant Pathogens. APSnet Education Center. The Plant Health Instructor. doi:10.1094/PHI-A-2004-0524-01

McDonald, B.A., Stukenbrock, E. H. (2016) Rapid emergence of pathogens in agro-ecosystems: global threats to agricultural sustainability and food security. Philosophical Transactions of the Royal Society of London B, Biological Sciences, 371, 20160026.

McMullen, M., Bergstrom, G., De Wolf, E., Dill-Macky, R., Hershman, D., Shaner, G. and Van Sanford, D. (2012) A unified effort to fight an enemy of wheat and barley: Fusarium head blight. Plant Disease, 96, 1712-1728.

Paini, D.R., Sheppard, A.W., Cook, D.C., De Barro, P.J., Worner, S.P. and Thomas, M.B. (2016) Global threat to 610 agriculture from invasive species. Proceedings of the National Academy of Sciences, 113, 7575-7579.

611 Roelfs, A.P. (1978) Estimated losses caused by rust in small grain cereals in the United States-1918-76. US 612 Department of Agriculture, Miscellaneous Publications, 1363, 85 pp. 
613 Roelfs, A.P. (1985) Wheat and Rye stem rust. In: Roelfs, A.P. Bushnell, W.R. (Eds.), The Cereal Rusts. Volume II:

614 Diseases Distribution, Epidemiology, and Control. Orlando, USA: Academic Press.

615 Saponari, M., Giampetruzzi, A., Loconsole, G., Boscia and D., Saldarelli, P. (2019) Xylella fastidiosa in olive in 616 Apulia: where we stand. Phytopathology, 109, 175-186.

617 Savary, S. and Willocquet, L. (2014) Simulation Modeling in Botanical Epidemiology and Crop Loss analysis.

618 APSnet Education Center. The Plant Health Instructor. doi: 10.1094/PHI-A-2014-0314-01.

619 Savary, S., Begaglio, S., Willocquet, L., Gustafson, D., Mason, D'Croz D., Sparks, A., et al. (2017) Crop health and 620 its global impacts on the components of food security. Food Security, 9, 311-327.

621 Savary, S., Willocquet, L., Pethybridge, S.J., Esker, P., McRoberts, N. and Nelson, A. (2019) The global burden of 622 pathogens and pests on major food crops. Nature Ecology \& Evolution, 3, 430-439.

623 Savary, S., Teng, P.S., Willocquet, L. and Nutter, F.W. Jr. (2006) Quantification and modeling of crop losses: a 624 review of purposes. Annual Review of Phytopathology, 44, 89-112.

625 Saunders, D.G., Pretorius, Z.A. and Hovmøller, M.S. (2019) Tackling the re-emergence of wheat stem rust in 626 Western Europe. Communications Biology, 2, 51.

627 Shaw, M.W. (1994) Seasonally induced chaotic dynamics and their implications in models of plant disease. Plant $628 \quad$ Pathology, 43, 790-801.

629 Singh, R. P., Hodson, D.P., Jin, Y., Lagudah, E.S., Ayliffe, M.A., Bhavani, S., et al. (2015) Emergence and spread of 630 new races of wheat stem rust fungus: continued threat to food security and prospects of genetic control. $631 \quad$ Phytopathology, 105, 872-884.

632 de Vallavieille-Pope, C., Bahri, B., Leconte, M., Zurfluh, O., Belaid, Y., Maghrebi, E., et al. (2018) Thermal 633 generalist behaviour of invasive Puccinia striiformis f. sp. tritici strains under current and future climate 634 conditions. Plant Pathology, 67, 1307-1320.

635 Van den Berg, F., Bacaer, N., Metz, J.A., Lannou, C. and van den Bosch, F. (2011) Periodic host absence can select 636 for higher or lower parasite transmission rates. Evolution Ecology, 25, 121-137. 
637 Wilcox, B.A. and Colwell, R.R. (2005) Emerging and reemerging infectious diseases: biocomplexity as an

638 interdisciplinary paradigm. EcoHealth, 2, 244-257.

639 Zadoks, J.C. (1974) The role of epidemiology in modern phytopathology. Phytopathology 64, 918-923.

640 Zadoks, J.C. and Schein, R.D. (1979) Epidemiology and Plant Disease Management. New York, USA: Oxford

$641 \quad$ University Press.

642 Zwankhuizen, M.J. and Zadoks, J.C. (2002) Phytophthora infestans's 10-year truce with Holland: a long-term

643 analysis of potato late-blight epidemics in the Netherlands. Plant Pathology, 51, 413-423.

644 
647 Assuming that the dynamics of the two pathogen strains are independent of each other, the simulation model 648 described in Sec. 2.2 [Eqs. (1)-(4)] can be summarised in a single equation:

$649 i_{j, t+1}=R\left(i_{j, t}\right) i_{j, t}$

650 Where

$651 R\left(i_{j, t}\right)=\frac{i m a x \times c o n v S P \times \exp \left[\left(R R g_{j} \times T_{S}\right)-\left(R R D_{j} \times T_{B S}\right)\right]}{i m a x+i_{j, t}\left[\exp \left(R R g_{j} \times T_{S}\right)-1\right]}$

Here, $i_{j, t}$ is the injury caused by the pathogen strain $j(j=1$ for the local strain, and $j=2$ for the immigrant strain) at

653 the very beginning of the growing season $t$, where $t$ is the index that runs through successive cropping cycles

654 (i.e., $t=1,2,3 \ldots)$. $T_{\mathrm{S}}$ is the duration of the growing season ( $\mathrm{CGP}$ ) and $T_{\mathrm{BS}}$ is the duration between two successive

655 growing seasons. Eq. (A1) is a map that relates the injury at the beginning of growing season $t+1, i_{\mathrm{j}, t+1}$, to the 656 injury at the beginning of the previous growing season $t, i_{\mathrm{j}, \mathrm{t}}$, representing a nonlinear generalisation of the 657 classical geometric growth model. The map Eq. (A1) has two fixed points:

$658 i_{j, F P 1}=0 ; i_{j, F P 2}=\frac{i m a x \times c o n v S P \times \exp \left[\left(R R g_{j} \times T_{S}\right)-\left(R R D_{j} \times T_{B S}\right)\right]-i m a x}{\exp \left(R R g_{j} \times T_{S}\right)-1}$

659 The fixed points determine the long-term outcomes of the dynamics: eventually the strain $j$ either dies out

660 (FP1) or reaches the stable equilibrium (FP2). The equilibrium occurs due to a balance between pathogen

661 reproduction during the growing season and its decay between growing seasons: the number of newly

662 produced pathogen individuals during the growing season compensates the number of individuals lost during

663 the preceding between-growing season phase. Which of the two fixed points is achieved in the long run, is

664 determined by the growth rate of the map Eq. (A1) linearised in the vicinity of FP1:

$665 R_{\mathrm{op}, \mathrm{j}}=\mathrm{convSP} \times \exp \left[\left(R R g_{\mathrm{j}} \times T_{\mathrm{s}}\right)-\left(R R D_{\mathrm{j}} \times T_{\mathrm{BS}}\right)\right]$ 
666 where $R_{0 p, j}$ quantifies the reproductive fitness of the strain $j$ and corresponds here to the polyetic basic

667 reproductive number. Usually, the basic reproductive number is defined as the number of host individuals that

668 are infected by a single infected host introduced into a fully susceptible host population (e.g., Zadoks and

669 Schein, 1979; Anderson and May, 1986; Campbell and Madden, 1990). Adapted to our context, the biological

670 meaning of $R_{0 \mathrm{p}, \mathrm{j}}$ is the number of units of crop injury appearing at the beginning of growing season $t+1$

671 following the introduction of a unit host injury at the beginning of the previous growing season $t$. If each of the

672 two strains is viable when present alone, i.e., $R_{0 \mathrm{p}, \mathrm{j}}>1$, the strain that has a higher basic reproductive number

673 eventually outcompetes the other strain. Consequently, the immigrant strain emerges if it has a higher polyetic

674 basic reproductive number, i.e., $R_{0 \mathrm{p}, 2}>R_{0 \mathrm{p}, 1}$. The emergence threshold is given by the equality of the two

675 polyetic basic reproductive numbers: $R_{0 \mathrm{p}, 2}=R_{0 \mathrm{p}, 1}$. We solve this equation with respect to $R R g_{2}$, using Eq. (A4), to

676 obtain the threshold value of $R R g_{2}$ above which the emergence takes place:

$677 R R g_{2, \text { thresh }}=\left(T_{\mathrm{BS}} / T_{S}\right) \times\left(R R d_{2}-R R d_{1}\right)+R R g_{1}$

678 Similarly, the emergence threshold can be expressed in terms of $R R D_{2}$ :

$679 R R D_{2, \text { thresh }}=\left(T_{S} / T_{B S}\right) \times\left(R R g_{2}-R R g_{1}\right)+R R D_{1}$

680 Here, the immigrant strain emerges when its relative decay rate is below the threshold, i.e., $R R D_{2}<R R D_{2, \text { thresh }}$.

681 When only one pathogen strain is present, $R_{0 p, j}$ given by Eq. (A4) determines without any approximation which

682 of the two fixed points in Eq. (A3) will be achieved according to the map in Eq. (A1). However, when both

683 pathogen strains are present, Eq. (A4) and Eqs. (A5), (A6) derived from it, give only approximate expressions for

684 emergence thresholds, under the assumption that the saturation effects of the logistic growth are negligible.

685 Nevertheless, $\mathrm{i}_{\mathrm{j}, \mathrm{FP} 2}$ in Eq. (A3) provides an exact expression for the final, equilibrium level of injury due to the 686 pathogen strain that wins the competition.

687 
Table 1. Description of the model variables

\begin{tabular}{|c|c|c|c|}
\hline Acronym & Dimension & Unit & Value \\
\hline \multicolumn{4}{|c|}{ State variables: } \\
\hline$i$ & $\begin{array}{l}\text { Injury caused by disease on a crop } \\
\text { stand }\end{array}$ & $\%$ & 0 to 100 \\
\hline$S$ & Number of surviving propagules & $\%$ & 0 to 100 \\
\hline$Y L$ & $\begin{array}{l}\text { Yield loss - yield reduction from a } \\
\text { disease-free attainable yield }\end{array}$ & $\%$ & 0 to 100 \\
\hline \multicolumn{4}{|l|}{ Rates: } \\
\hline$R P I$ & Rate of primary infections $\quad\left[\mathrm{T}^{-1}\right]$ & $\%$ Day $^{-1}$ & \\
\hline RconvlS & $\begin{array}{l}\text { Rate of conversion from injury (i) } \\
\text { into surviving propagules (S) }\end{array}$ & $\%$ Day $^{-1}$ & \\
\hline Rdecay & $\begin{array}{l}\text { Rate of decay of surviving } \\
\text { propagules }\end{array}$ & $\%$ Day $^{-1}$ & \\
\hline resetYL & $\begin{array}{l}\text { Rate of reset of yield loss at each } \\
\text { cropping cycle }\end{array}$ & $\%$ Day $^{-1}$ & \\
\hline RI & Rate of injury increase & $\%$ Day $^{-1}$ & \\
\hline$R L$ & Rate of yield loss & $\%$ Day $^{-1}$ & \\
\hline$R M$ & $\begin{array}{l}\text { Rate of immigration of the } \\
\text { pathogen }\end{array}$ & $\%$ Day $^{-1}$ & \\
\hline starter & $\begin{array}{l}\text { Rate of infection to initiate } \\
\text { epidemics }\end{array}$ & $\%$ Day $^{-1}$ & \\
\hline \multicolumn{4}{|c|}{ Parameters: } \\
\hline CEP & $\begin{array}{l}\text { Duration of the crop establishment }[\mathrm{T}] \\
\text { period }\end{array}$ & Day & 120 \\
\hline CGP & Duration of the crop growth period $[\mathrm{T}]$ & Day & 120 \\
\hline convSP & $\begin{array}{l}\text { Conversion of surviving propagules }\left[\mathrm{T}^{-1}\right] \\
\text { into a rate of primary infections }\end{array}$ & Day $^{-1}$ & 0.01 \\
\hline imax & $\begin{array}{l}\text { Carrying capacity of injury - } \\
\text { maximum level of injury }\end{array}$ & $\%$ & 100 \\
\hline$R R D_{\mathrm{j}}$ & $\begin{array}{l}\text { Relative rate of decay of surviving } \quad\left[\mathrm{T}^{-1}\right] \\
\text { propagules for strain } \mathrm{j}\end{array}$ & Day $^{-1}$ & $\begin{array}{l}j=1: 0.01 \\
j=2: \text { Varying }\end{array}$ \\
\hline$R R g_{\mathrm{j}}$ & $\begin{array}{l}\text { Relative rate of epidemic (injury) } \\
\text { increase for strain } \mathrm{j}\end{array}$ & Day $^{-1}$ & $\begin{array}{l}j=1: 0.07 \\
j=2: \text { Varying }\end{array}$ \\
\hline$R R L$ & Relative rate of yield loss & Day $^{-1}$ & 0.05 \\
\hline$Y a$ & $\begin{array}{l}\text { Attainable yield - uninjured yield } \\
\text { level }\end{array}$ & $\%$ & 100 \\
\hline
\end{tabular}




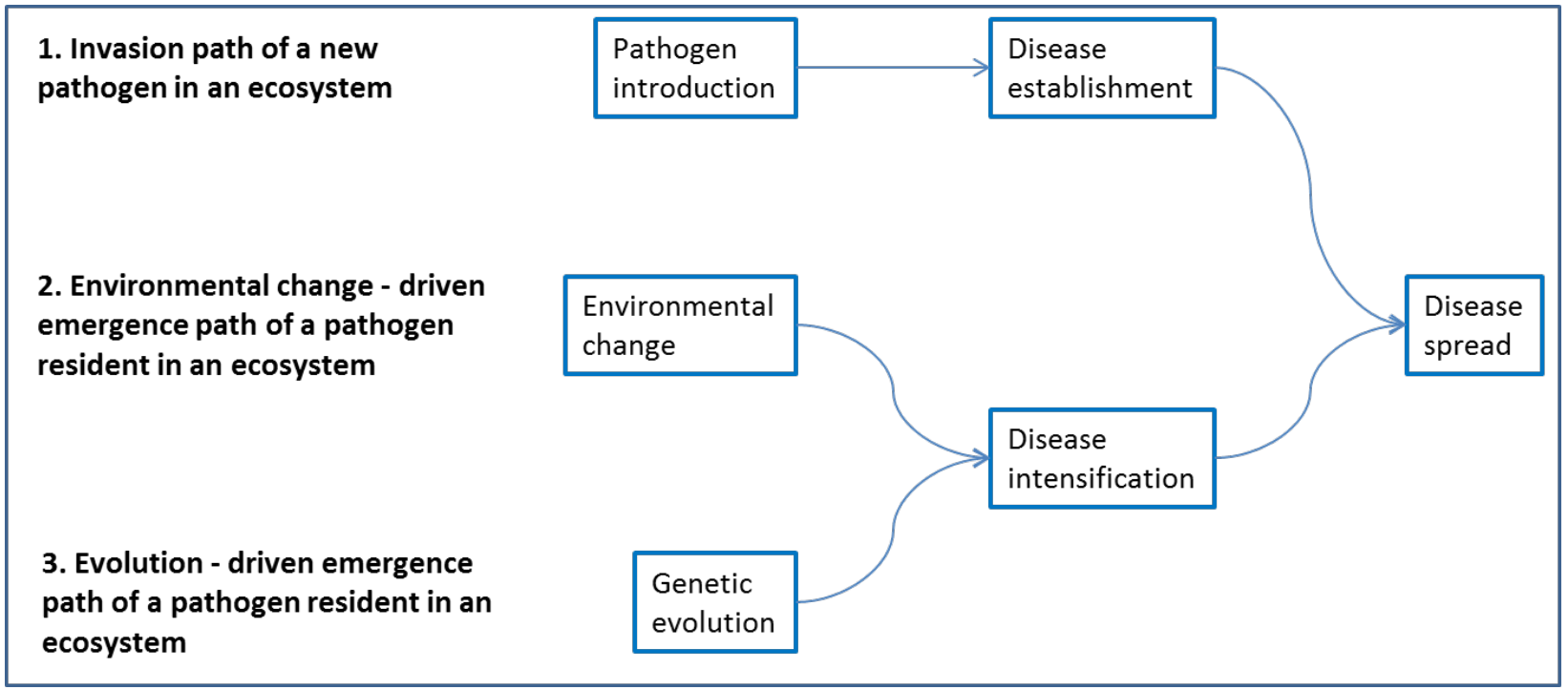

Fig. 1. A framework for analysis of emerging epidemics: paths and processes. Three paths for emergence are considered (left, bold characters), involving different processes (in boxes). Paths may be combined, e.g., paths 1 and 3, involving both introduction and evolution, or 2 and 3, involving environmental change and evolution. See text for examples. 


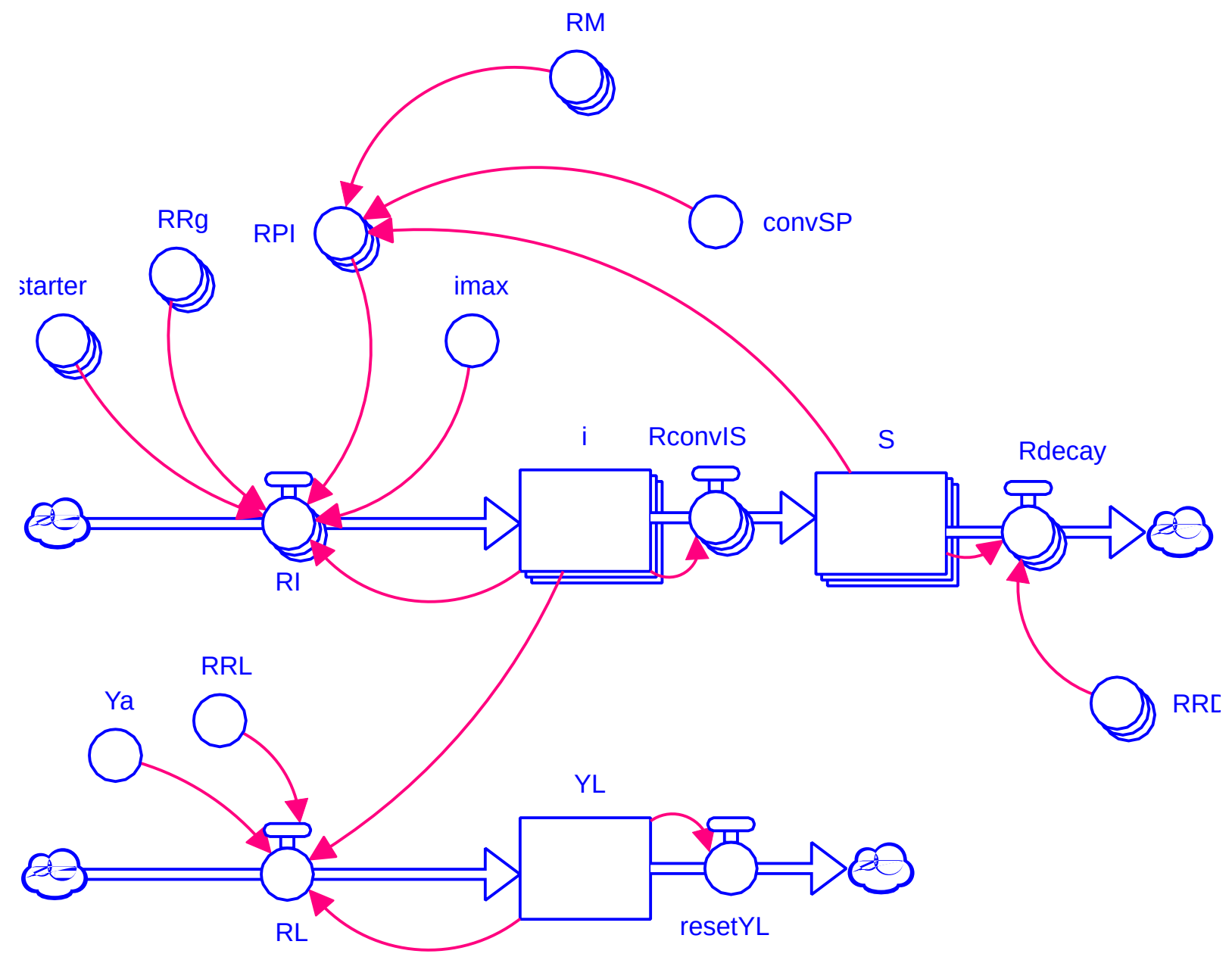

Fig. 2. Simplified flowchart of the process-based model used to analyse emerging diseases of crop plants. Variable acronyms are described in Table 1. The flowchart uses symbols introduced by J Forrester (Forrester, 1961): rectangles represent state variables; valves represent rates of change of state variables; circles represent parameters or computed variables. Stacked symbols (e.g., state variables) represent vectors of two pathogen strains. 


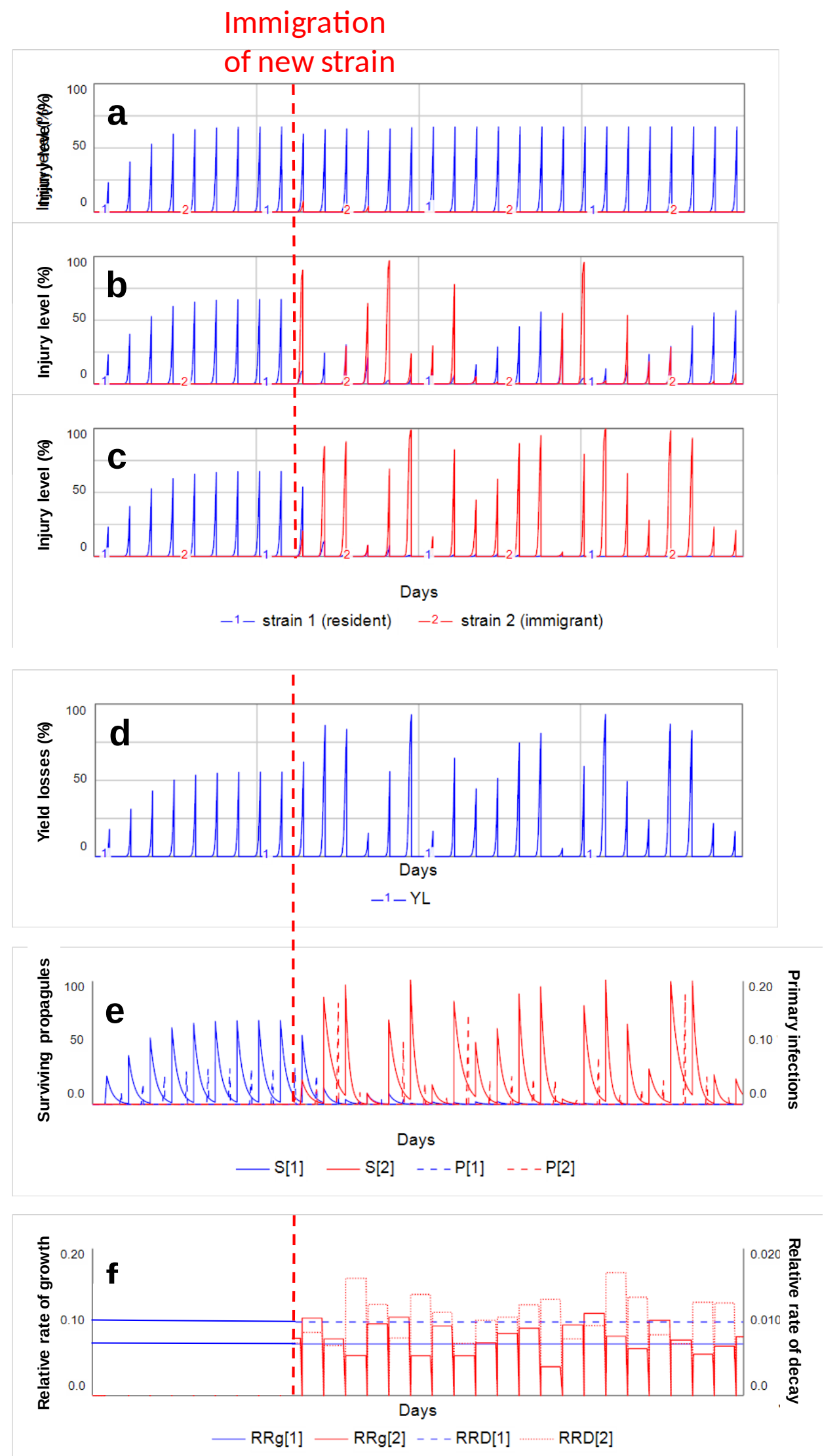

Fig. 3. Examples of simulated dynamics of injury levels and related variables over 30 cropping cycles. 
(a) to (c): simulated injury levels from different runs where $R R g_{2} \sim N(0.07,0.03)$ and $R R D_{2} \sim N(0.01$, 0003). (a): a simulation where the immigrant strain did not emerge; (b): a simulation where the immigrant and resident strains compete over cropping cycles; (c): a simulation where the immigrant strain replaces the resident strain; (d) to (f): simulated dynamics of other variables, associated with the dynamic of injury levels shown in (c).

Except for $R R g_{2}$ and $R R D_{2}$, all parameters are set according to Table 1. Immigration of strain 2 takes place at the end of the crop establishment period of cropping cycle 10, while strain 1 is established at the beginning of the simulation. 

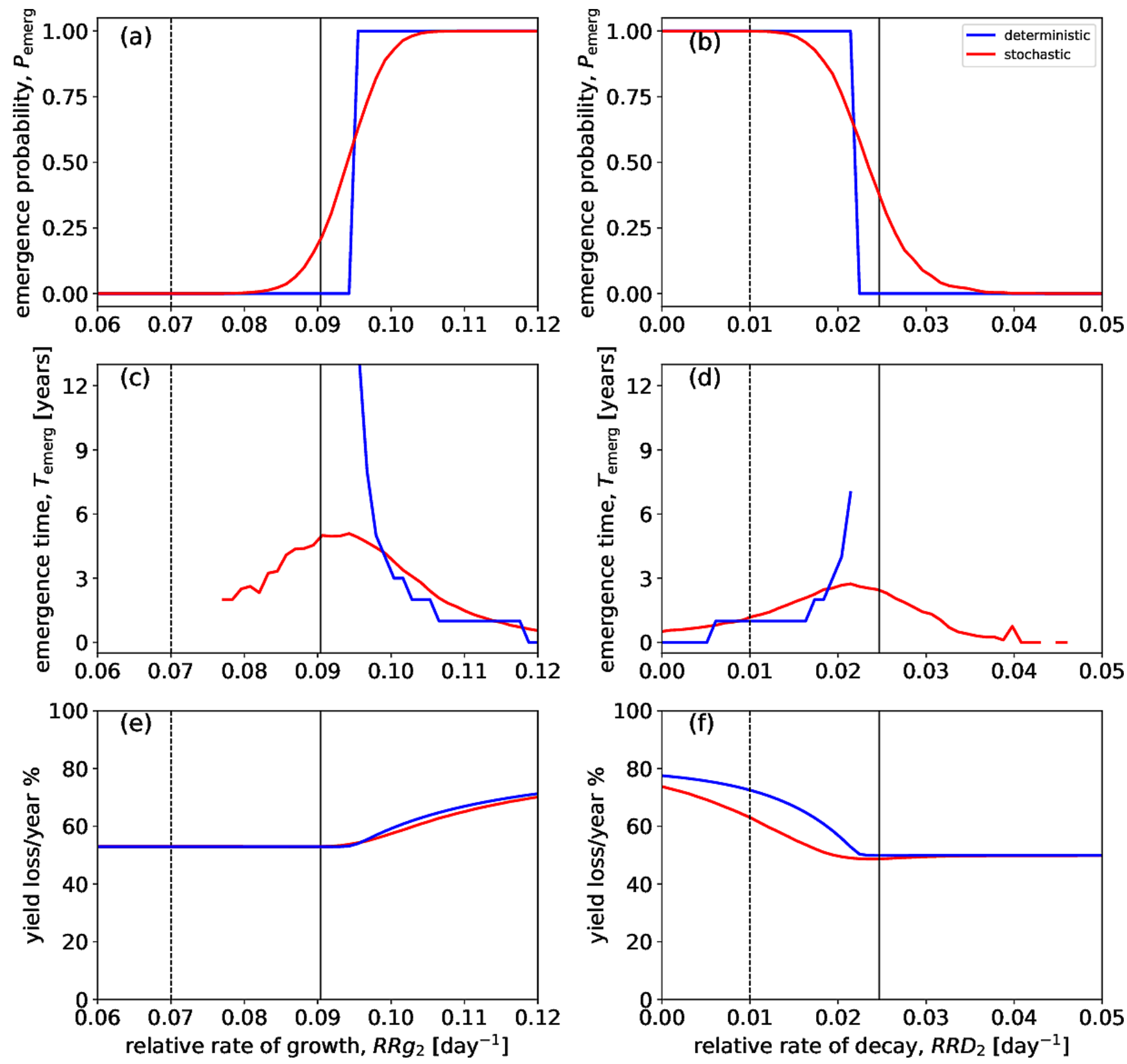

Fig. 4. Effects of pathogen reproduction and survival parameters on disease emergence and yield loss.

Emergence probabilities (a), (b), emergence times (c), (d) and yield losses (e), (f) are plotted versus the relative rate of growth, $R R g_{2}(\mathrm{a}),(\mathrm{c}),(\mathrm{e})$, and the relative rate of decay, $R R D_{2}(\mathrm{~b}),(\mathrm{d}),(\mathrm{f})$, of the immigrant strain. Blue curves correspond to the deterministic regime, in which both $R R g_{2}$ and $R R D_{2}$ have fixed, deterministic values, whereas red curves correspond to the stochastic regime. Red curves in panels (a), (c), (e) were computed with the values of $R R D_{2}$ drawn as random numbers from the normal (Gaussian) distribution at the beginning of each cropping cycle with the mean 0.02 day $^{-1}$ and the standard deviation 0.007 day $^{-1}$, while $R R g_{2}$ 
assumed fixed, deterministic values. Similarly, red curves in panels (b), (d), (f) were computed with the values of $R R g_{2}$ drawn as random numbers from the normal (Gaussian) distribution at the beginning of each cropping cycle with the mean 0.1 day $^{-1}$ and the standard deviation 0.035 day $^{-1}$, while $R R D_{2}$ assumed fixed, deterministic values.

Dashed vertical lines indicate the value of the relative rate of growth, $R R g_{1}=0.07$, of the resident strain in (a), (c), (e), and the value of the relative rate of decay, $R R D_{1}=0.01$, of the resident strain in (b), (d), (f). Solid vertical lines show the approximate analytical emergence thresholds, according to Eq. (6) in (a), (c), (e) and Eq. (7) in (b), (d), (f). Values of other parameters are given in Table 1. 

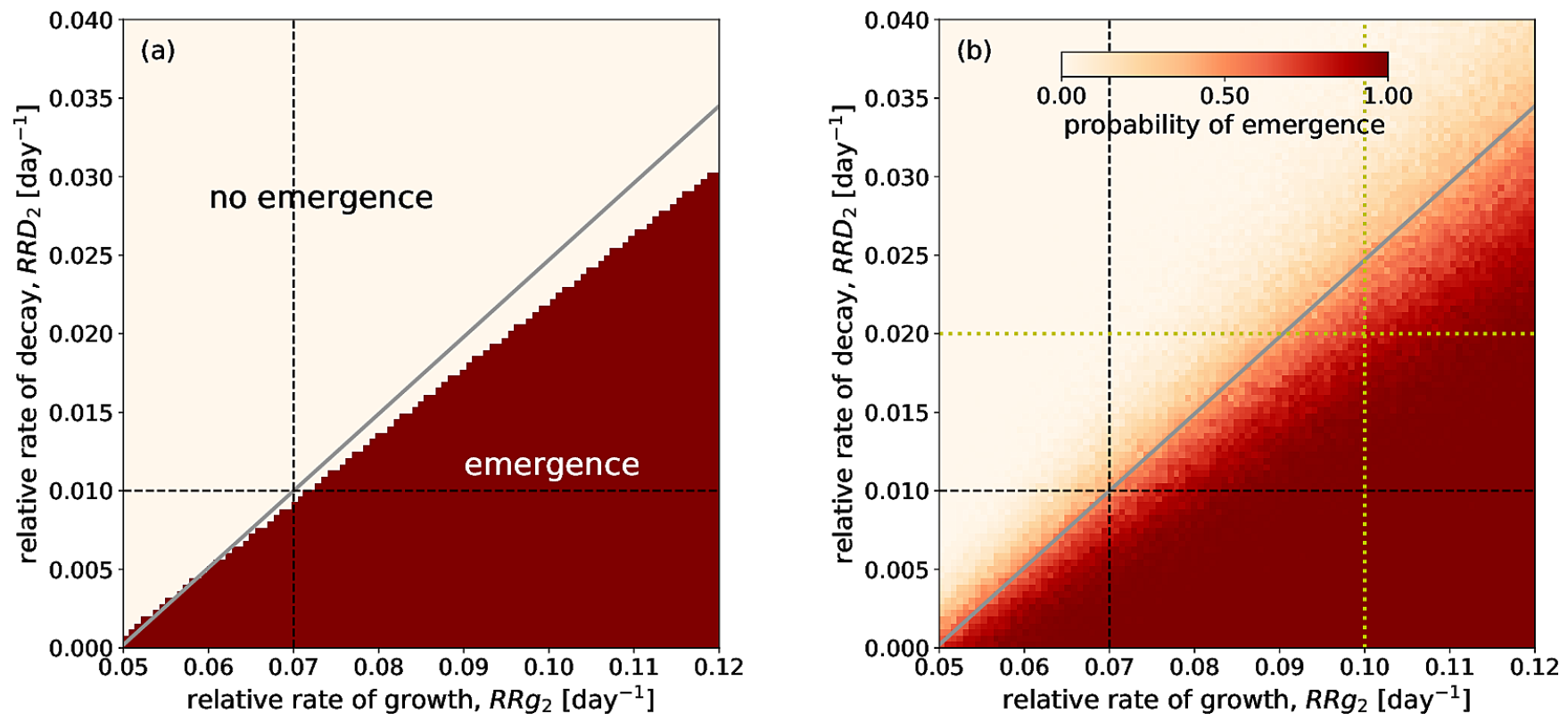

Fig. 5. Combined effects of pathogen reproduction and survival parameters on probability of emergence.

(a) deterministic regime; (b) stochastic regime, whereby the values of $R R g_{2}$ and $R R D_{2}$ were drawn from the normal (Gaussian) distribution at the beginning of each cropping cycle with the mean corresponding to the values on $\mathrm{x}$ - and $\mathrm{y}$-axes and the standard deviation constituting a constant proportion, 0.35 , of the corresponding mean values. Values of other parameters are given in Table 1.

In each panel, the grey diagonal line shows the analytical threshold according to Eq. (7). Dashed black lines indicate the fitness values of the resident strain: vertical $R R g_{1}=0.07$ day $^{-1}$; horizontal $R R D_{1}=0.01$ day $^{-1}$. Dashed yellow lines mark the parameter regions explored in Fig. 4: $R R D_{2}=0.02$ day $^{-1}$ in Fig. $4 \mathrm{a}$, c, e; $R R g_{2}=0.1$ day $^{-1}$ in Fig. $4 b, d, f$. 


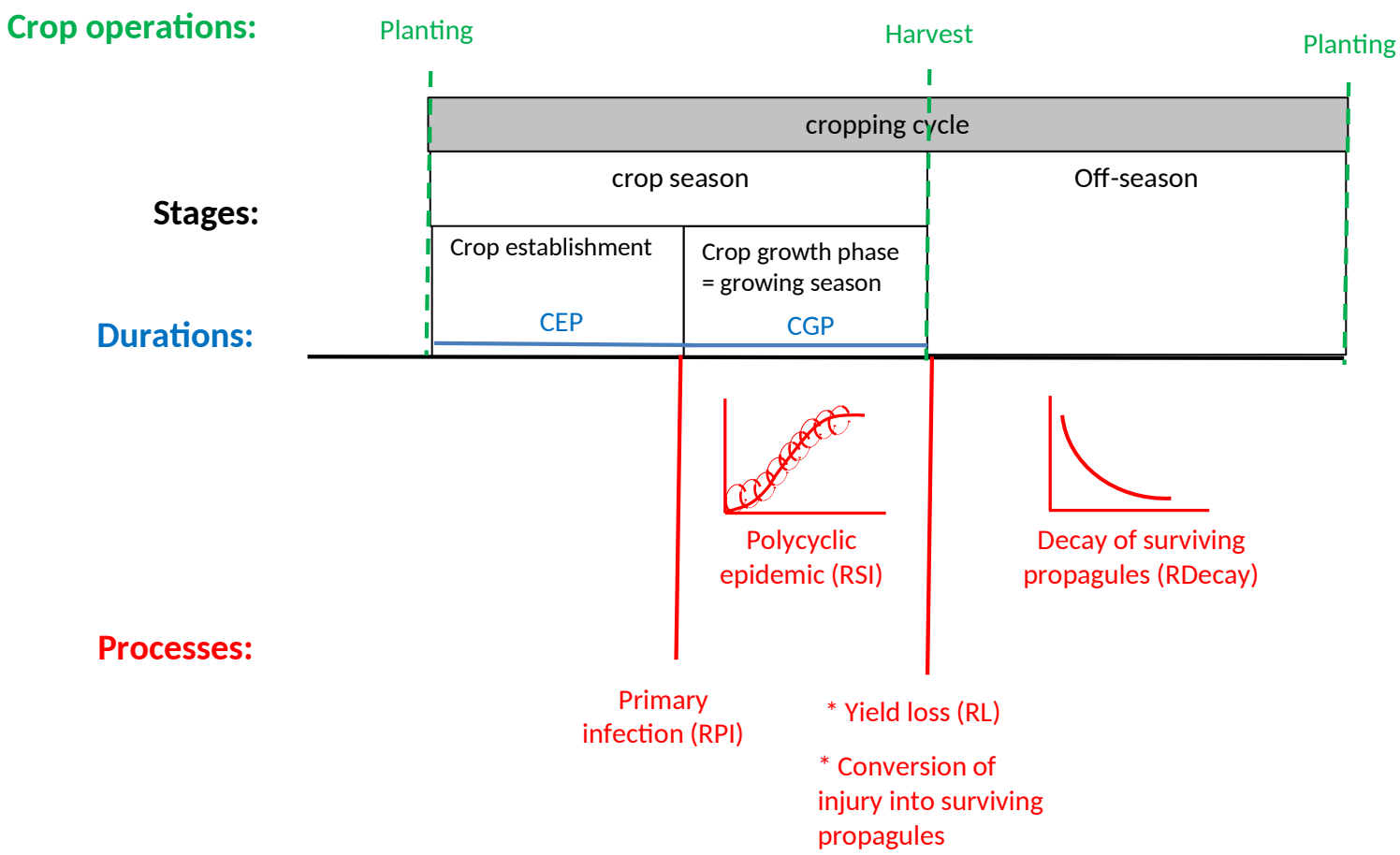

Supplementary Figure 1. Main stages and processes considered in the polyetic model at each cropping cycle. CEP: duration of the Crop Establishment Phase; CGP: duration of Crop Growth phase. 